\title{
THE SOLUTION OF THE SKEWED PLATE
}

\author{
By Dr. Eng., Kitami Okamoto, C.E. Member*
}

Synopsis : The author searched the solution of the skewed plate with two opposite edges simply supported and the other edges free by the method of Fourier's transformation and this solution can apply to the other skewed plate with two opposite edges simply supported and the other edges various conditions.

\section{The general solution of rectangular plate}

The deflection $\zeta(x, y)$ of the middle surface of a thin homogeneous isotropic plate must satisfy the following differential equation over the region bounded by the plate in Fig.1.

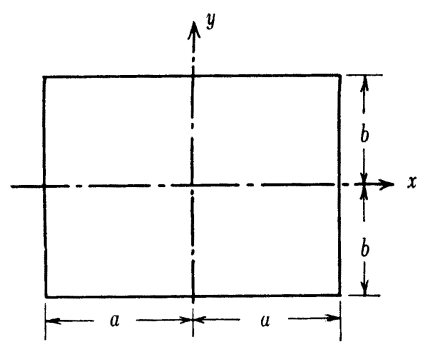

Fig. 1 Rectangular plate and system of coordinates.

$$
\frac{\partial^{4} \zeta}{\partial x^{4}}+2 \frac{\partial^{4} \zeta}{\partial x^{2} y^{2}}+\frac{\partial^{4} \zeta}{\partial y^{4}}=\frac{p(x y)}{D}
$$

where $p(x, y)=$ load per unit area normal to surface of plate.

$$
\begin{aligned}
& D=\frac{E h^{3}}{12\left(1-\nu^{2}\right)}, \quad h=\text { thickness of plate } \\
& E=\text { young's modulus } \\
& \nu=\text { poisson's ratio of material }
\end{aligned}
$$

We assume that a solution of Equation (1) may be written is the form.

$$
\zeta=\zeta_{0}+\zeta_{1}
$$

where

$\zeta_{0}=\mathrm{a}$ particular solution decided by load functions.

$\zeta_{1}=\mathrm{a}$ general solution decided by boundary conditions of rectangular plate.

A general solution $\zeta_{1}$ consist in potential function of the form.

$$
\varphi_{0}(x y)=f(x+i y)+g(x-i y)
$$

And bi-potential function in the form $x \varphi_{1}$.

* Civil Engineering Research Institute of Hokkaido Development Bureau. $(x, y)$ or $y \varphi_{2}(x, y)$. In the case of full uniform load the equation (2) is expressed in the form.

$$
\begin{aligned}
\zeta= & \frac{p b^{4}}{24 D}\left(\frac{y^{4}}{b^{4}}-6 \frac{y^{2}}{b^{2}}+5\right)+f_{1}(x+i y)+g_{1}(x-i y) \\
& +x\left\{f_{2}(x+i y)+g_{2}(x-i y)\right\} \cdots \cdots \cdots \cdots \cdots(3)
\end{aligned}
$$

\section{The solution of the skewed plate.}

In Fig. 2, the solution of the skewed plate is expressed from Equation (3) in the form.

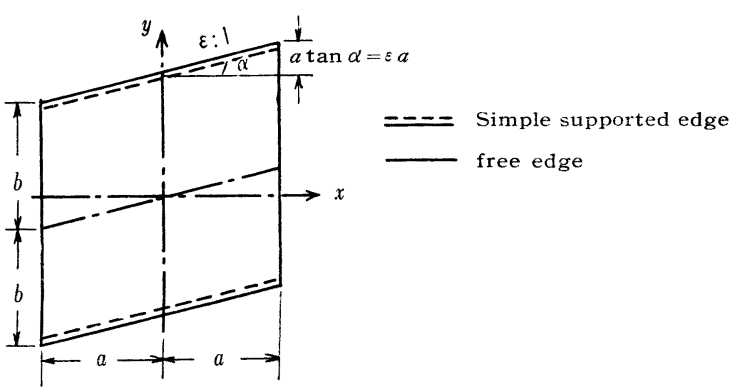

Fig. 2 The Skewed plate with two opposite edges simply supported and the other edges-free.

$$
\begin{aligned}
\zeta= & \frac{p}{24 D\left(1+\varepsilon^{2}\right)^{2}}\left\{(y-\varepsilon x)^{4} \frac{1}{b^{4}}-6 \frac{1}{b^{2}}(y-\varepsilon x)^{2}\right. \\
& +5\}+g_{1}(x-i y)+f_{1}(x+i y)+(x+\varepsilon y) \\
& \cdot\left\{\frac{1}{1+i \frac{\varepsilon}{\varepsilon}} f_{2}(x+i y)+\frac{1}{1-i \varepsilon} g_{2}(x-i y)\right\}
\end{aligned}
$$

The equation must satisfy the following boundary conditions at the sides $y=\varepsilon x+b$.

$$
\zeta=0, \quad \frac{\delta^{2} \zeta}{\partial y^{2}}+\frac{\delta^{2} \zeta}{\partial x^{2}}=0
$$

Substituting the condition (5) in the equation (4), we obtain the following equation as result of calculation,

$$
\begin{aligned}
\zeta= & \frac{p}{24 D\left(1+\varepsilon^{2}\right)^{2}}\left\{(y-\varepsilon x)^{4}-6 b^{2}(y-\varepsilon x)^{2}\right. \\
& \left.+5 b^{4}\right\} \frac{1}{b^{4}}+\left[2 \frac{(x+\varepsilon y)}{b} \varepsilon \sum_{n=2,4,6 \cdots} C_{n} \cdot \sin \frac{n \pi}{2 b}(\varepsilon x\right. \\
& -y) \cosh \frac{n \pi}{2 b}(x+\varepsilon y)+2 \frac{(x+\varepsilon y)}{b} \sum_{n=1,3,5 \ldots} D_{n} \\
& \cdot \cos \frac{n \pi}{2 b}(\varepsilon x-y) \cdot \sinh \frac{n \pi}{2 b}(x+\varepsilon y)-\sum_{n=2,4,6} A_{n} \\
& \cdot \sin \frac{n \pi}{2 b}(\varepsilon x-y) \sinh \frac{n \pi}{2 b}(\varepsilon y+x)-\sum_{n=1,3,5 \cdots} B_{n} \\
& \left.\cdot \cos \frac{n \pi}{2 b}(\varepsilon x-y) \cdot \cosh \frac{n \pi}{2 b}(x+\varepsilon y)\right] \\
& \frac{1}{\cosh \frac{n \pi}{2} \varepsilon\left(a^{\prime}+1\right)} \cdot \cdots \cdots \cdots \ldots \ldots \ldots \ldots \ldots \ldots(6)
\end{aligned}
$$


The constants of integration $A_{n}, B_{n}, C_{n}, D_{n}$, can be determined from the boundary conditions on the sides $x= \pm a$.

\section{The analysis of the skewed plate}

The boundary conditions of the skewed plate on the sides $x= \pm a$ are

$$
\begin{aligned}
& \left|\frac{\partial^{2} \zeta}{\partial x^{2}}+\nu \frac{\partial^{2} \zeta}{\partial y^{2}}\right|_{x= \pm a}=0 \\
& \left|\frac{\partial^{3} \zeta}{\partial x^{3}}+(2-\nu) \frac{\partial^{3} \zeta}{\partial x \partial y^{2}}\right|_{x= \pm a}=0
\end{aligned}
$$

Appling the conditions (7) to the equation (6), we obtain the following equations.

$$
\begin{aligned}
& \sum_{n=2,4,6 \cdots} g_{n} C_{n}+\sum_{n=1,3,5 \ldots} h_{n} D_{n}+\sum_{n=2,4,6 \cdots} i_{n} A_{n}+\sum_{n=1,3,5 \cdots} j_{n} B_{n}=R(y) \\
& \underset{n=2,4,6, \cdots}{g_{n}{ }^{\prime} C_{n}}+\sum_{n=1,3,5, \cdots} h_{n}{ }^{\prime} D_{n}+\sum_{n=2,4,6, \ldots} i_{n}{ }^{\prime} A_{n}+\sum_{n=1,3,5, \cdots} j_{n}{ }^{\prime} B_{n}=R^{\prime}(y)
\end{aligned}
$$

where

$$
\begin{aligned}
g_{n}= & \frac{n}{\pi}\left\{\varepsilon^{2}(1-\nu) f_{n}{ }^{(a)}+\varepsilon\left(1+\nu \varepsilon^{2}\right) \phi_{n}{ }^{(a)}\right\} \\
& +\frac{1}{2}(1-\nu) \varepsilon^{2}\left(a^{\prime}-y^{\prime}\right) n^{2}\left\{\varphi_{n}{ }^{(a)}\left(1-\varepsilon^{2}\right)\right. \\
& +2 \varepsilon \tau_{n^{(a)}}{ }^{(a)} \\
h_{n}= & 2 \frac{n}{\pi}\left\{\left(1+\nu \varepsilon^{2}\right) f_{n}^{(a)}-\varepsilon(1-\nu) \phi_{n}{ }^{(a)}\right\} \\
& +\frac{1}{2}(1-\nu) \varepsilon\left(a^{\prime}-y^{\prime}\right) n^{2}\left\{\tau_{n}{ }^{(a)}\left(1-\varepsilon^{2}\right)\right. \\
& \left.-2 \varepsilon \varphi_{n}{ }^{(a)}\right\} \\
j_{n}= & -\frac{n^{2}}{4}(1-\nu)\left\{f_{n}{ }^{(a)}\left(1-\varepsilon^{2}\right)-2 \varepsilon \phi_{n}{ }^{(a)}\right\}, \\
i_{n}= & -\frac{n^{2}}{4} \varepsilon(1-\nu)\left\{\phi_{n}^{(a)}\left(1-\varepsilon^{2}\right)+2 \varepsilon f_{n}{ }^{(a)}\right\}, \\
R\left(y^{\prime}\right)= & -\frac{\left(y^{\prime 2}-1\right)}{2 \pi^{2}\left(1+\varepsilon^{2}\right)^{2}}\left(\nu+\varepsilon^{2}\right) \ldots \cdots \cdots \cdots \cdots
\end{aligned}
$$

and

$$
\begin{aligned}
g_{n}{ }^{\prime}= & \frac{n^{2}}{\pi}\left[\left\{\varepsilon(1-\nu)\left(\varepsilon^{3}-2 \varepsilon\right)+\varepsilon^{2}\left(1+\varepsilon^{2}\right)\right\} \tau_{n}{ }^{(a)}\right. \\
& \left.+\left\{\varepsilon\left(1+\varepsilon^{2}\right)+\frac{1}{2}(1-\nu)\left(5 \varepsilon^{2}-1\right) \varepsilon\right\} \varphi_{n}{ }^{(a)}\right] \\
& +\frac{n^{3}}{4}\left(a^{\prime}-y^{\prime}\right)(1-\nu) \varepsilon^{2}\left\{f_{n}^{(a)}\left(\varepsilon^{3}-3 \varepsilon\right)\right. \\
& \left.+\phi_{n}^{(a)}\left(3 \varepsilon^{2}-1\right)\right\} \\
h_{n}{ }^{\prime}= & \frac{n^{2}}{n}\left[\left\{(1-\nu)\left(2 \varepsilon-\varepsilon^{3}\right)-\varepsilon\left(1+\varepsilon^{2}\right)\right\} \varphi_{n}{ }^{(a)}\right. \\
& \left.+\left\{\left(1+\varepsilon^{2}\right)+\frac{1}{2}(1-\nu)\left(5 \varepsilon^{2}-1\right)\right\} \tau_{n}{ }^{(a)}\right] \\
& +\frac{n^{3}}{4}\left(a^{\prime}-y^{\prime}\right)(1-\nu) \varepsilon\left\{\left(3 \varepsilon^{2}-1\right) f_{n}{ }^{(a)}\right. \\
& \left.-\left(\varepsilon^{3}-3 \varepsilon\right) \phi_{n}^{(a)}\right\} \\
j_{n}{ }^{\prime}= & -(1-\nu) \frac{n^{3}}{8}\left\{\tau_{n}{ }^{(a)}\left(3 \varepsilon^{2}-1\right)-\varphi_{n}{ }^{(a)}\left(\varepsilon^{3}-3 \varepsilon\right)\right\} \\
i_{n}{ }^{\prime}= & -(1-\nu) \frac{n^{3}}{8} \varepsilon\left\{\tau_{n}^{(a)}\left(\varepsilon^{3}-3 \varepsilon\right)+\varphi_{n}^{(a)}\left(3 \varepsilon^{2}-1\right)\right\} \\
R_{n}{ }^{\prime}= & -\frac{\varepsilon}{\pi^{3}\left(1+\varepsilon^{2}\right)}\left(1+\frac{1-\nu}{1+\varepsilon^{2}}\right) y^{\prime}
\end{aligned}
$$

Writing $\quad y^{\prime}=\varepsilon a-y, \quad a^{\prime}=\left(\varepsilon+\frac{1}{\varepsilon}\right) \frac{a}{b}$,

In the expression $(7)^{\prime}(8)^{\prime}$, the value of $\varphi_{n}{ }^{(a)}$, $f_{n}{ }^{(a)}, \tau_{n}{ }^{(a)}, \phi_{n}{ }^{(a)}$, are these of $\varphi_{n}, \tau_{n}, f_{n}, \phi_{n}$ in $x=a$.

where

$$
\begin{aligned}
\varphi_{n}= & \sin \frac{n \pi}{2 b}\left(\varepsilon x-y^{\prime}\right) \quad \cosh \frac{n \pi}{2 b}(x+\varepsilon y), \\
& \frac{1}{\cosh \frac{n \pi}{2} \varepsilon\left(a^{\prime}+1\right)} \\
\tau_{n}= & \cos \frac{n \pi}{2 b}(\varepsilon x-y) \sinh \frac{n \pi}{2 b}(x+\varepsilon y), \\
& \frac{1}{\cosh \frac{n \pi}{2} \varepsilon\left(a^{\prime}+1\right)} \\
\phi_{n}= & \sin \frac{n \pi}{2 b}(\varepsilon x-y) \sinh \frac{n \pi}{2 b}(x+\varepsilon y), \\
& \frac{\cosh \frac{n \pi}{2} \varepsilon\left(a^{\prime}+1\right)}{\cos \frac{n \pi}{2} \varepsilon\left(a^{\prime}+1\right)} \\
f_{n}= & \cos \frac{n \pi}{2 b}(\varepsilon x-y) \cosh \frac{n \pi}{2 b}(x+\varepsilon y),
\end{aligned}
$$

From the expression (10), these values are represented by Fourier Sine series for $0 \leqq y \leqq 1$ as follows.

$$
\begin{aligned}
& \text { i.e. } \varphi_{n}{ }^{(a)}=\sum_{s=1}^{\infty} \varphi_{n s^{(1)}} \sin s \pi y=\sum_{s=1}^{\infty}\left(\vartheta^{\prime}{ }_{n s}{ }^{(1)}\right. \\
& \left.-\vartheta_{n s}{ }^{(1)}\right) \sin s \pi y \\
& \tau_{n}{ }^{(a)}=\sum_{s=1}^{\infty} \tau_{n s}{ }^{(1)} \sin s \pi y=\sum_{s=1}^{\infty}\left(w_{n s}{ }^{(1)}\right. \\
& \left.-w^{\prime} n s^{(1)}\right) \sin s \pi y \\
& \phi_{n}{ }^{(a)}=\sum_{s=1}^{\infty} \phi_{n s}{ }^{(1)} \sin s \pi y=\sum_{s=1}^{\infty}\left(z^{\prime}{ }_{n s}^{(1)}\right. \\
& -z_{\left.n s^{(1)}\right) \sin s \pi y} \\
& f_{n}{ }^{(a)}=\sum_{s=1}^{\infty} f_{n s}{ }^{(1)} \sin s \pi y=\sum_{s=1}^{\infty}\left(u_{n s}{ }^{(1)}\right. \\
& \left.-u_{n s}^{\prime}{ }^{(1)}\right) \sin s \pi y \\
& y f_{n}{ }^{(a)}=\sum_{n=1}^{\infty} f^{\prime}{ }_{n s}^{(1)} \sin s \pi y \\
& -\sum_{s=1}^{\infty} f_{n s}{ }^{(2)} \sin s \pi y \\
& y \varphi_{n}^{(a)}=\sum_{n=1}^{\infty} \varphi^{\prime}{ }_{n s}^{(1)} \sin s \pi y \\
& -\sum_{s=1}^{\infty} \varphi_{n s}{ }^{(2)} \sin s \pi y \\
& y \tau_{n}{ }^{(a)}=\sum_{n-1}^{\infty} \tau^{\prime}{ }_{n s}^{(1)} \sin s \pi y \\
& -\sum_{s=1}^{\infty} \tau_{n s}{ }^{(2)} \sin s \pi y \\
& y \phi_{n}{ }^{(a)}=\sum_{n=1}^{\infty}{\phi^{\prime}}_{n s^{(1)}} \sin s \pi y
\end{aligned}
$$




\section{$-\sum_{s=1}^{\infty} \phi_{n t}{ }^{(2)} \sin s \pi y$}

where

$$
\begin{aligned}
& f_{n s^{\prime(1)}}=\left(\bar{u}_{n s^{(1)}}-\bar{u}_{n s^{(1)}}\right), \\
& f_{n s^{(2)}}=\left(u_{n s^{(2)}}-u_{n s^{(2)}}\right), \cdots \cdots \cdots \text { etc. }
\end{aligned}
$$

the value of $\bar{u}_{n s}{ }^{(1)}, \bar{u}_{n s}{ }^{\prime(1)}, \cdots .$. etc. is the value of the expression (12) substituting $a_{n s}{ }^{\prime}, b_{n s}$ ', $c_{n s}^{\prime}, d_{n s}^{\prime}$, instead of $a_{n s}, b_{n s}, c_{n s}, d_{n s}$, writing $\quad y=\frac{1}{2}\left(1+y^{\prime}\right) . \quad(0 \leqq y \leqq 1)$

The new symbols of $u_{n s}{ }^{(1)}, u_{n s}{ }^{(2)}, \vartheta_{n s}{ }^{(1)}, \vartheta_{n s}{ }^{(2)}$, ......etc are noted as follows.

$$
\begin{aligned}
& u_{n s^{(1)}}=\frac{1}{\pi \lambda_{n s}}\left\{n \varepsilon a_{n s}-(n+s) b_{n s}\right\} \\
& u_{n s}^{\prime}{ }^{(1)}=\frac{1}{\pi \lambda^{\prime} n s}\left\{n \varepsilon a_{n s}-(n-s) b_{n s}\right\} \\
& \vartheta_{n s^{(1)}}=\frac{1}{\pi \lambda_{n s}}\left\{n \varepsilon c_{n s}+(n+s) d_{n s}\right\} \\
& \vartheta_{n s^{\prime(1)}}=\frac{1}{\pi \lambda^{\prime} n}\left\{n \varepsilon c_{n s}+(n-s) d_{n s}\right\} \\
& w_{n s^{(1)}}=\frac{1}{\pi \lambda_{n s}}\left\{-n \varepsilon d_{n s}+(n+s) c_{n s}\right\} \\
& w_{n s^{\prime}}^{(1)}=\frac{1}{\pi \lambda^{\prime}}\left\{-n \varepsilon d_{n s}+(n-s) c_{n s}\right\} \\
& z_{n s}^{(1)}=\frac{-1}{\pi \lambda_{n s}}\left\{n \varepsilon b_{n s}+(n+s) a_{n s}\right\} \\
& z_{n s}^{\prime(1)}=\frac{-1}{\pi \lambda^{\prime}}\left\{n \varepsilon b_{n s}+(n-s) a_{n s}\right\}
\end{aligned}
$$

and

$$
\begin{aligned}
& u_{n s}^{(2)}=\frac{-1}{\pi \lambda_{n s}}\left\{n \varepsilon w_{n s}^{(1)}+(n+s) \vartheta_{n s}^{(1)}\right\}, \\
& u_{n s^{\prime}}{ }^{(2)}=\frac{-1}{\pi \lambda^{\prime}{ }_{n s}}\left\{n \varepsilon w^{(1)}+(n-s) \vartheta^{\prime}{ }_{n s}^{(1)}\right\} \text {, } \\
& \vartheta_{n s}{ }^{(2)}=\frac{1}{\pi \lambda_{n s}}\left\{-n \varepsilon z_{n s}{ }^{(1)}+(n+s) u_{n s^{(1)}}\right\} \text {, } \\
& \vartheta_{n s^{\prime(2)}}=\frac{1}{\pi \lambda^{\prime} n s}\left\{-u \varepsilon z^{\prime}{ }_{n s}^{(1)}+(n-s) u_{n s^{\prime}}^{(1)}\right\} \text {, } \\
& w_{n s^{(2)}}=\frac{-1}{\pi \lambda_{n s}}\left\{n \in u_{n s^{(1)}}+(n+s) z_{n s^{(1)}}\right\}, \\
& w_{n s^{\prime(2)}}=\frac{-1}{\pi \lambda^{\prime}{ }_{n s}}\left\{n \varepsilon u^{\prime}{ }_{n s}^{(1)}+(n-s){z^{\prime}}_{n s^{(1)}}\right\} \text {, } \\
& z_{n s}^{(2)}=\frac{1}{\pi \lambda_{n s}}\left\{-n \varepsilon \vartheta_{n s^{(1)}}+(n+s) w_{n s^{(1)}}\right\} \text {, } \\
& z_{n s^{\prime(2)}}=\frac{1}{\pi \lambda^{\prime}{ }_{n s}}\left\{-n \varepsilon \vartheta^{\prime}{ }_{n s^{(1)}}+(n-s) w^{\prime}{ }_{n s^{(1)}}\right\} \text {, }
\end{aligned}
$$

writing

$$
\begin{aligned}
\lambda_{n s}= & n^{2} \varepsilon^{2}+(n+s)^{2}, \lambda^{\prime}{ }_{n s}=n^{2} \varepsilon^{2}+(n-s)^{2}, \\
a_{n s}= & \frac{-(-1)^{\frac{n-1}{2}}}{\cosh \frac{n \pi}{2} \varepsilon\left(a^{\prime}+1\right)}\left\{(-1)^{s} \sinh \frac{n \pi}{2} \varepsilon\left(a^{\prime}-1\right)\right. \\
& \left.+\sinh \frac{n \pi}{2} \varepsilon\left(a^{\prime}+1\right)\right\}
\end{aligned}
$$

$$
\begin{aligned}
b_{n s}= & \frac{(-1)^{\frac{n}{2}}}{\cosh \frac{n \pi}{2} \varepsilon\left(a^{\prime}+1\right)}\left\{(-1)^{s} \cosh \frac{n \pi}{2} \varepsilon\left(a^{\prime}-1\right)\right. \\
& \left.-\cosh \frac{n \pi}{2} \varepsilon\left(a^{\prime}+1\right)\right\} \\
c_{n s}= & \frac{-(-1)^{\frac{n}{2}}}{\cosh \frac{n \pi}{2} \varepsilon\left(a^{\prime}+1\right)}\left\{(-1)^{s} \sinh \frac{n \pi}{2} \varepsilon\left(a^{\prime}-1\right)\right. \\
& \left.-\sinh \frac{n \pi}{2} \varepsilon\left(a^{\prime}+1\right)\right\} \\
d_{n s}= & \frac{(-1)^{\frac{n-1}{2}}}{\cosh \frac{n \pi}{2} \varepsilon\left(a^{\prime}+1\right)}\left\{(-1)^{s} \cosh \frac{n \pi}{2} \varepsilon\left(a^{\prime}-1\right)\right. \\
& \left.+\cosh \frac{n \pi}{2} \varepsilon\left(a^{\prime}+1\right)\right\} \\
d_{n s}^{\prime}= & \frac{(-1)^{\frac{n-1}{2}}}{\cosh \frac{n \pi}{2} \varepsilon\left(a^{\prime}+1\right)}(-1)^{s} \cosh \frac{n \pi}{2} \varepsilon\left(a^{\prime}-1\right), \\
a_{n s}^{\prime}= & \frac{-(-1)^{\frac{n-1}{2}}}{\cosh \frac{n \pi}{2} \varepsilon\left(a^{\prime}+1\right)}\left\{(-1)^{s} \sinh \frac{n \pi}{2} \varepsilon\left(a^{\prime}-1\right)\right\}, \\
c_{n s}^{\prime}= & \frac{-(-1)^{\frac{n}{2}}}{\cosh \frac{n \pi}{2} \varepsilon\left(a^{\prime}+1\right)}(-1)^{s} \sinh \frac{n \pi}{2} \varepsilon\left(a^{\prime}-1\right), \\
b_{n s}^{\prime}= & \frac{(-1)^{\frac{n}{2}}}{\cosh \frac{n \pi}{2} \varepsilon\left(a^{\prime}+1\right)}(-1)^{s} \cosh \frac{n \pi}{2} \varepsilon\left(a^{\prime}-1\right),
\end{aligned}
$$

and then, expanding Equation (8), (9) to Fourier Sine series, each value of every terms in expression (8)' are expressed as follows.

$$
\begin{aligned}
& g_{n s}=2 \frac{n}{\pi}\left\{\varepsilon^{2}(1-\nu) f_{n s^{(1)}}+\varepsilon\left(1+\nu \varepsilon^{2}\right) \phi_{n s}{ }^{(1)}\right\} \\
& +\frac{1}{2}(1-\nu) \varepsilon^{2} n^{2}\left[( a ^ { \prime } + 1 ) \left\{\left(1-\varepsilon^{2}\right) \varphi_{n s^{(1)}}\right.\right. \\
& \left.+2 \varepsilon \tau_{n s}{ }^{(1)}\right\}-2\left\{\left(1-\varepsilon^{2}\right)\left(\varphi_{n s^{(1)}}-\varphi_{n s^{(2)}}\right)\right. \\
& \left.+2 \varepsilon\left(\tau^{\prime} n s^{(1)}-\tau_{n s^{(2)}}\right\}\right] \\
& h_{n s}=2 \frac{n}{\pi}\left\{\left(1+\nu \varepsilon^{2}\right) f_{n s}{ }^{(1)}-\varepsilon(1-\nu) \phi_{n s^{(1)}}\right\} \\
& +\frac{1}{2}(1-\nu) \varepsilon n^{2}\left[( a ^ { \prime } + 1 ) \left\{\left(1-\varepsilon^{2}\right) \tau_{n s}^{(1)}\right.\right. \\
& \left.-2 \varepsilon \varphi_{n s^{(1)}}\right\}-2\left\{\left(1-\varepsilon^{2}\right)\left(\tau_{n s^{\prime(1)}}-\tau_{n s^{(2)}}\right)\right. \\
& \left.\left.-2 \varepsilon\left(\varphi^{\prime}{ }_{n s}^{(1)}-\varphi_{n s^{(2)}}\right)\right\}\right] \\
& j_{n s}=-\frac{n^{2}}{4}(1-\nu)\left\{\left(1-\varepsilon^{2}\right) f_{n s}^{(1)}-2 \varepsilon \phi_{n s^{(1)}}\right\} \\
& i_{n s}=-\frac{n^{2}}{4}(1-\nu) \varepsilon\left\{\left(1-\varepsilon^{2}\right) \phi_{n s^{(1)}}+2 \varepsilon f_{n s^{(1)}}\right\} \\
& R_{s}=\frac{8\left(\nu+\varepsilon^{2}\right)}{\pi^{5} s^{3}\left(1+\varepsilon^{2}\right)^{2}}\left\{1-(-1)^{s}\right\}
\end{aligned}
$$

and

$$
\begin{aligned}
g_{n s}^{\prime}= & \frac{n^{2}}{\pi} \varepsilon\left(c_{1} \tau_{n s^{(1)}}+c_{2} \varphi_{n s^{(1)}}\right)+\frac{n^{3}}{4} \varepsilon^{2}\left[\left(a^{\prime}+1\right)\right. \\
& \cdot\left(c_{4} f_{n s^{(1)}}+c_{3} \phi_{n s^{(1)}}\right)-2\left\{c _ { 4 } \left(f_{n s^{\prime(1)}}\right.\right.
\end{aligned}
$$




$$
\begin{aligned}
& \left.\left.\left.-f_{n s^{(2)}}\right)+c_{3}\left(\phi_{n s^{(1)}}-\phi_{n s^{(2)}}\right)\right\}\right] \\
& h^{\prime}{ }_{n s}=\frac{n^{2}}{\pi}\left(-c_{1} \varphi_{n s}{ }^{(1)}+c_{2} \tau_{n s^{(1)}}\right)+\frac{n^{3}}{4} \varepsilon\left[\left(a^{\prime}+1\right)\right. \\
& \text { - }\left(c_{3} f_{n s}{ }^{(1)}-c_{4} \phi_{n s}{ }^{(1)}\right)-2\left\{c _ { 3 } \left(f_{n s^{\prime}}{ }^{(1)}\right.\right. \\
& \left.\left.\left.-f_{n s}{ }^{(2)}\right)-c_{4}\left(\phi_{n s^{(1)}}-\phi_{n s}{ }^{(2)}\right)\right\}\right] \\
& j^{\prime}{ }_{n s}=-\frac{n^{3}}{8}\left(c_{3} \tau_{n s}{ }^{(1)}-c_{4} \varphi_{n s}{ }^{(1)}\right) \\
& i^{\prime}{ }_{n s}=-\frac{n^{3}}{8} \varepsilon\left(c_{4} \tau_{n s}^{(1)}+c_{3} \varphi_{n s}{ }^{(1)}\right) \\
& R_{s}{ }^{\prime}=\frac{2}{\pi^{3}} \cdot \frac{\varepsilon}{1+\varepsilon^{3}}\left(1+\frac{1-\nu}{1+\varepsilon^{2}}\right) \frac{1+(-1)^{s}}{s \pi}
\end{aligned}
$$

where

$$
\begin{aligned}
& c_{1}=\varepsilon\left(1+\varepsilon^{2}\right)-(1-\nu)\left(2 \varepsilon-\varepsilon^{3}\right), \\
& c_{2}=1+\varepsilon^{2}+\frac{1}{2}(1-\nu)\left(5 \varepsilon^{2}-1\right), \\
& c_{3}=(1-\nu)\left(3 \varepsilon^{2}-1\right), \quad c_{4}=(1-\nu)\left(\varepsilon^{3}-3 \nu\right)
\end{aligned}
$$

Thus, we have two groups of equations providing relation for determining the unknown constants $A_{n}, C_{n}, B_{n}$, and $D_{n}$. Furthermore, the value of $\sinh \frac{n \pi}{2} \varepsilon\left(a^{\prime}-1\right)$ or $\cosh \frac{n \pi}{2} \varepsilon\left(a^{\prime}-1\right)$ becomes negligible in comparison with $\sinh \frac{n \pi}{2}$ - $\varepsilon\left(a^{\prime}+1\right)$ when $n \rightarrow \infty$. In that case, the value of $f_{n s}, \tau_{n s}, \phi_{n s}, \varphi_{n s}$, is expressed as follows.

$$
\begin{aligned}
& \underset{n=1,3,5, \cdots}{f_{n s}^{(1)}}=\underset{\substack{n s \\
n=1,3,5, \cdots}}{\tau_{n s}^{(1)}}=W_{n s}{ }^{(1)} \frac{a_{n s}}{\pi}, \\
& \underset{n=1,3,5, \ldots}{\phi_{n s}{ }^{(1)}}=\underset{n=1,3,5, \cdots}{\varphi_{n s}{ }^{(1)}}=V_{n s}{ }^{(1)} \frac{a_{n s}}{\pi} \\
& \underset{n=1,3,5, \cdots}{f_{n s}^{(2)}}=\underset{\substack{n s \\
n=1,3,5, \ldots}}{\tau^{(2)}}=W_{n s}{ }^{(2)} \frac{a_{n s}}{\pi^{2}}, \\
& \underset{n=1,3,5, \cdots}{\phi_{n s}{ }^{(2)}}=\underset{n=1,3,5,}{\varphi_{n s}{ }^{(2)}}=V_{n s}{ }^{(2)} \frac{a_{n s}}{\pi^{2}},
\end{aligned}
$$

and

$$
\begin{aligned}
& \underset{n=2,4,6, \cdots}{f_{n s}{ }^{(1)}}=\underset{n=2,4,6, \cdots}{\tau_{n s}{ }^{(1)}}=-V_{n s}{ }^{(1)} \frac{b_{n s}}{\pi}, \\
& \underset{n=2,4,6, \ldots}{\phi_{n s}^{(1)}}=\underset{n=2,4,6, \ldots}{\varphi_{n s}^{(1)}}=W_{n s}^{(1)} \frac{b_{n s}}{\pi}, \\
& \underset{n=2,4,6, \cdots}{f_{n s^{(2)}}=\tau_{n s}{ }_{n=2,4,6, \ldots}^{(2)}=-V_{n s}{ }^{(2)}} \frac{b_{n s}}{\pi^{2}}, \\
& \underset{n=2,4,6, \ldots}{\phi_{n s}{ }^{(2)}}=\underset{n=2,4,6, \ldots}{\varphi_{n s}^{(2)}}=W_{n s}{ }^{(2)} \frac{b_{n s}}{\pi^{2}},
\end{aligned}
$$

where when $n \gg s$, the value of $W_{n s}, V_{n s}$ is expressed as follows.

$$
\begin{aligned}
& W_{n s}^{(1)}=-\frac{4 \varepsilon s}{n^{2}\left(1+\varepsilon^{2}\right)^{2}}, \quad V_{n s}{ }^{(1)}=--\frac{2\left(1-\varepsilon^{2}\right) s}{n^{2}\left(1+\varepsilon^{2}\right)^{2}}, \\
& W_{n s}{ }^{(2)}=\frac{4 s\left(3 \varepsilon^{2}-1\right)}{n^{3}\left(1+\varepsilon^{2}\right)^{3}}, \quad V_{n s}{ }^{(2)}=\frac{4 s \varepsilon\left(3-\varepsilon^{2}\right)}{n^{3}\left(1+\varepsilon^{2}\right)^{3}}
\end{aligned}
$$

Applying the expression (18) to the expression (16),

$$
\begin{aligned}
& j_{n}=0, \quad i_{n}=0, \quad g_{n}=-\frac{8 \varepsilon^{2} s}{n \pi^{2}\left(1+\varepsilon^{2}\right)}(-1)^{n / 2}, \\
& h_{n}=-\frac{8 \varepsilon s}{n \pi^{2}\left(1+\varepsilon^{2}\right)}(-1)^{n / 2},
\end{aligned}
$$

Similary applying to the expression (17),

$$
\begin{aligned}
& j_{n}{ }^{\prime}=\frac{1}{4} \varepsilon(1-\nu) n \frac{s}{\pi}(-1)^{\frac{n-1}{2}}, \\
& i_{n}{ }^{\prime}=\frac{1}{4} \varepsilon^{2}(1-\nu) n \frac{s}{\pi}(-1)^{n / 2}, \\
& h_{n}{ }^{\prime}=\left\{\frac{2 \varepsilon}{\pi}+\frac{1}{2} \varepsilon^{2} n(1-\nu)\left(a^{\prime}+1\right)\right\} \frac{s}{\pi}(-1)^{\frac{n-1}{2}}, \\
& g_{n}{ }^{\prime}=\left\{\frac{2 \varepsilon}{\pi}+\frac{1}{2} \varepsilon^{2} n(1-\nu)\left(a^{\prime}+1\right)\right\} \frac{s}{\pi} \varepsilon(-1)^{\frac{n-1}{2}},
\end{aligned}
$$

From the expression $(19)_{b}$, we obtain the following results.

$$
\begin{aligned}
\text { i.e. } \quad h^{\prime}{ }_{n s} & =\frac{s}{s+1} h^{\prime}{ }_{n s+1}, & g^{\prime}{ }_{n s} & =\frac{s}{s+1} g^{\prime}{ }_{n s+1}, \\
j^{\prime}{ }_{n s} & =\frac{s}{s+1} j^{\prime}{ }_{n s+1}, & i_{n s}^{\prime} & =\frac{s}{s+1} i_{n s+1}^{\prime},
\end{aligned}
$$

From the expression $(19)_{c}$, the expression (17) can be transformed as follows.

i.e. $\quad \bar{h}^{\prime}{ }_{n s}=\frac{s}{s-1} h^{\prime}{ }_{n s-1}-h^{\prime}{ }_{n s}$,

$$
\begin{gathered}
\bar{g}_{n s}^{\prime}=\frac{s}{s-1} g^{\prime}{ }_{n s-1}-g^{\prime}{ }_{n s}, \\
\bar{j}_{n s}^{\prime}=\frac{s}{s-1} j^{\prime}{ }_{n s-1}-j^{\prime}{ }_{n s}, \\
\bar{i}_{n s}^{\prime}=\frac{s}{s-1} i^{\prime}{ }_{n s-1}-i_{n s}^{\prime}, \\
\text { for } s \geqq 2
\end{gathered}
$$

In the case of $s=1$

$$
\begin{aligned}
& {\overline{h^{\prime}}}_{n_{1}}=\int_{0}^{1} h_{n} d y-h_{n_{1}}, \\
& \bar{g}^{\prime}{ }_{n_{1}}=\int_{0}^{1} g_{n} d y-g^{\prime}{ }_{n_{1}}, \\
& \bar{j}^{\prime}{ }_{n_{1}}=\int_{0}^{1} j_{n}^{\prime} d y-j^{\prime}{ }_{n_{1}} \\
& \bar{i}_{n_{1}}^{\prime}=\int_{0}^{1} i_{n} d y-i_{n_{1}},
\end{aligned}
$$

where

$$
\begin{aligned}
& \int_{0}^{1} h_{n} d y=-\frac{\varepsilon}{\pi}\left[\frac{2}{\pi}\left(1+r_{2}\right)+\frac{1}{2}(1-\nu) \varepsilon n\right. \\
& \text { - } \left.\left\{\left(a^{\prime}+1\right)-\frac{2 r_{1}}{\alpha_{1}+\gamma_{1}}\right\}\left(\alpha_{1}+r_{1}\right)\right](-1)^{\frac{n-1}{2}} \text {, } \\
& \int_{0}^{1} g_{n} d y=-\frac{\varepsilon}{\pi}\left[\frac{2 \varepsilon}{\pi}\left(\alpha_{1}-\gamma_{1}\right)+\frac{1}{2}(1-\nu) \varepsilon^{2} n\right. \\
& \text { - } \left.\left\{\left(a^{\prime}+1\right)+\frac{2 r_{2}}{1-r_{2}}\right\}\left(1-r_{2}\right)\right](-1)^{n / 2}, \\
& \int_{0}^{1} j_{n} d y=\frac{n}{4}(1-\nu) \frac{\varepsilon}{\pi}\left(1+\gamma_{2}\right)(-1)^{\frac{n-1}{2}} \text {, } \\
& \int_{0}^{1} i_{n} d y=\frac{n}{4}(1-\nu) \frac{\varepsilon^{2}}{\pi}\left(\alpha_{1}-\gamma_{1}\right)(-1)^{n / 2}, \\
& \text { and } \quad r_{1}=\frac{\sinh \frac{n \pi}{2} \varepsilon\left(a^{\prime}-1\right)}{\cosh \frac{n \pi}{2} \varepsilon\left(a^{\prime}+1\right)}, \quad r_{2}=\frac{\cosh \frac{n \pi}{2} \varepsilon\left(a^{\prime}-1\right)}{\cosh \frac{n \pi}{2} \varepsilon\left(a^{\prime}+1\right)},
\end{aligned}
$$




$$
\alpha_{1}=\frac{\sinh \frac{n \pi}{2} \varepsilon\left(a^{\prime}+1\right)}{\cosh \frac{n \pi}{2} \varepsilon\left(a^{\prime}+1\right)},
$$

Finally we obtain following two groups of equations from the expression (16), (20).

i.e.

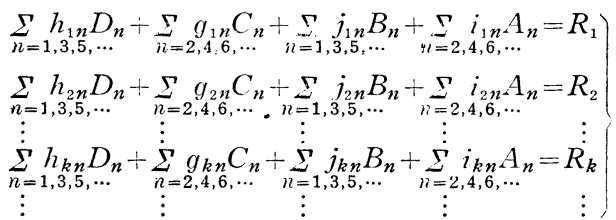

and

$\sum_{n=1,3,5, \cdots} \overline{h^{\prime}}{ }_{n} D_{n}+\sum_{n=2,4,6, \cdots} \overline{g^{\prime}}{ }_{n} C_{n}+\sum_{n=1,3,5, \ldots} \overline{j^{\prime}}{ }_{1 n} B_{n}+\sum_{n=2,4,6, \cdots} \overline{i_{1}^{\prime}}{ }_{1 n} A_{n}=R_{1}$ $\sum_{n=1,3,5, \ldots} \overline{h^{\prime}}{ }_{2 n} D_{n}+\sum_{n=2,4,6, \ldots} \overline{g^{\prime}}{ }_{2 n} C_{n}+\sum_{n=1,3,5, \ldots} \overline{j_{2 n}^{\prime}} B_{n}+\sum_{n=2,4,6, \ldots} \overline{i^{\prime}}{ }_{2 n} A_{n}=R_{2}^{\prime}$

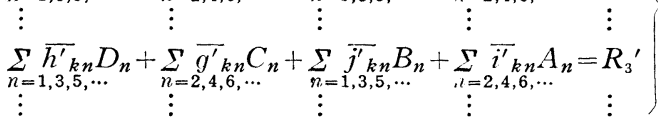

where two growps of these equations have special relations each other from the expression $(13)_{a},(18)_{b}$ and $(18)_{c}$. i.e. these equations are written as follows.

$$
\begin{aligned}
& \sum_{n=1,3,5, \cdots k-1} h_{1 n} D_{n}+\sum_{n=2,4,6, \cdots k-2} g_{1 n} C_{n}+\sum_{n=1,3,5, \cdots k-1} j_{1 n} B_{n}+\sum_{n=2,4,6, \cdots k-2} i_{1 n} A_{n} \\
& \quad+h_{1 k}\left(C_{k}+\alpha_{1 \cdot k+1} D_{k+1}+\alpha_{1 \cdot k+2} C_{k+2}+\cdots \cdots\right) \\
& \quad+j_{1 k}\left(A_{k}+\beta_{1 \cdot k+1} B_{k+1}+\beta_{1 \cdot k+2} A_{k+2} \cdots \cdots\right)=R_{1}
\end{aligned}
$$

in $k$ th equation

$$
\begin{aligned}
& \sum_{n=1,3,5, \cdots k-1} h_{k n} D_{n}+\sum_{n=2,4,6, \cdots k-2} g_{k n} C_{n}+\sum_{n=1,3,5, \cdots k-1} j_{k n} B_{n}+\sum_{n=2,4,6, \cdots k-2}^{i_{k n} A_{n}} \\
& \quad+h_{k k}\left(C_{k}+\alpha_{k k+1} D_{k+1}+\alpha_{k \cdot k+2} C_{k+2}+\cdots \cdots\right) \\
& \quad+j_{k k}\left(A_{k}+\beta_{k \cdot k+1} B_{k+1}+\beta_{k \cdot k+2} A_{k+2}+\cdots \cdots\right)=R_{k}
\end{aligned}
$$

Similary

$\sum_{n=1} \bar{h}^{\prime}{ }_{1 n} D_{n}+\sum_{n=2} \bar{g}^{\prime}{ }_{1 n} C_{n}+\sum_{n=1,3} \tilde{j}_{1 n}^{\prime} B_{n}+\sum_{n=2, \bar{i}_{1 n}}{ }_{1 n} A_{n}$

$$
\begin{aligned}
& +\bar{h}^{\prime}{ }_{1 k}\left(C_{k}+\alpha^{\prime}{ }_{1 \cdot k+1} D_{k+1}+\alpha^{\prime}{ }_{1 \cdot k+2} C_{k+2}+\cdots \cdots\right) \\
& \bar{j}^{\prime}{ }_{1 k}\left(A_{k}+\beta^{\prime}{ }_{1 \cdot k+1} B_{k+1}+\beta^{\prime}{ }_{1 \cdot k+2} A_{k+2}+\cdots \cdots\right)=R_{1}{ }^{\prime}
\end{aligned}
$$

in $k$ th equation

$$
\begin{aligned}
& \sum_{n=1,3,5, \cdots k-1} \bar{h}_{k n}^{\prime} D_{n}+\sum_{n=2,4,6, \cdots k-2}{\overline{g^{\prime}}}_{k n} C_{n}+\sum_{n=1,3,5, \cdots k-1} \bar{j}^{\prime}{ }_{k n} B_{n}+\sum_{n=2,4,6, \cdots k-2} \bar{i}_{k n}{ }_{k n} A_{n} \\
& +\bar{h}^{\prime}{ }_{k k}\left(C_{k}+\alpha^{\prime}{ }_{k \cdot k+1} D_{k+1}+\alpha^{\prime}{ }_{k \cdot k+2} C_{k+2}+\cdots \cdots\right) \\
& +\bar{j}^{\prime}{ }_{k k}\left(A_{k}+\beta^{\prime}{ }_{k \cdot k+1} B_{k+1}+\beta^{\prime}{ }_{k \cdot k+2} A_{k+2}+\cdots \cdots\right)=R^{\prime}{ }_{k}
\end{aligned}
$$

where each coefficient of unknown constant have approximately the following relations.

i.e. in the $k+t$ the coefficients.

$$
\begin{aligned}
& \alpha_{1 \cdot k+t} \fallingdotseq \alpha_{2 \cdot k+t} \fallingdotseq \alpha_{3 \cdot k+t} \cdots \cdots \fallingdotseq \alpha_{k \cdot k+t} \cdots \cdots \\
& \quad \fallingdotseq \alpha_{1}{ }_{k+t} \fallingdotseq \alpha_{2}{ }^{\prime}{ }_{k+t} \cdots \cdots \fallingdotseq \alpha^{\prime}{ }_{k \cdot k+t} \cdots \cdots(t=1,2,3, \cdots)
\end{aligned}
$$

and

$$
\begin{gathered}
\beta_{1 \cdot k+t} \fallingdotseq \beta_{2 \cdot k+t} \fallingdotseq \beta_{3 \cdot k+t} \cdots \cdots \fallingdotseq \beta_{k \cdot k+t} \cdots \cdots \\
\fallingdotseq \beta^{\prime}{ }_{1 \cdot k \cdot t} \fallingdotseq \beta^{\prime}{ }_{2 \cdot k+t} \cdots \cdots \fallingdotseq \beta^{\prime}{ }_{k \cdot k+t}
\end{gathered}
$$

By these relations, we can select the most properble and balanced value of each coefficient, where the value $\gamma_{t}$ and $r_{t}^{\prime}$ equals nearly each coefficients, $\alpha_{k \cdot k+t}, \alpha_{k \cdot k+t}^{\prime}$, and $\beta_{k \cdot k+t}, \beta^{\prime}{ }_{k \cdot k+t}$.

Therefore, unkown constants group containing these coefficient $\gamma_{t}, \gamma_{t}{ }^{\prime}$, are expressed as follows.

$$
\begin{aligned}
& \bar{C}_{2}=C_{2}+r_{3} D_{3}+\gamma_{4} C_{4}+r_{5} D_{5}+\cdots \cdots r_{k} C_{k}+\cdots \cdots \\
& \bar{A}_{2}=A_{2}+r_{3}{ }^{\prime} D_{3}+r_{4}{ }^{\prime} A_{4}+r_{5}{ }^{\prime} B_{5}+\cdots \cdots r_{k}{ }^{\prime} A_{k}+\cdots \cdots \\
& \bar{D}_{3}=D_{3}+\frac{1}{\gamma_{3}}\left(r_{4} C_{4}+r_{5} D_{5}+r_{6} C_{6}+\cdots \cdots r_{k} C_{k}+\cdots \cdots\right) \\
& \bar{B}_{3}=B_{3}+\frac{1}{\gamma_{3}{ }^{\prime}}\left(r_{4}{ }^{\prime} A_{4}+r_{5}{ }^{\prime} B_{5}+r_{6}{ }^{\prime} A_{6}+\cdots \gamma_{k}{ }^{\prime} A_{k}+\cdots\right)
\end{aligned}
$$

in the $k$ th unknown constant group

$$
\begin{aligned}
& \bar{C}_{k}=C_{k}+\frac{1}{\gamma_{k}}\left(\gamma_{k+1} D_{k+1}+\gamma_{k+2} C_{k+2}+\gamma_{k+3} D_{k+3} \cdots \cdots\right) \\
& \bar{A}_{k}=A_{k}+\frac{1}{\gamma^{\prime}{ }_{k}}\left(r^{\prime}{ }_{k+1} B_{k+1}+r^{\prime}{ }_{k+2} A_{k+2}\right. \\
& \left.+r^{\prime}{ }_{k+3} B_{k+3}+\cdots \cdots\right) \quad k \geqq 4
\end{aligned}
$$

Furthermore, from the expression (23)

$$
\begin{aligned}
& \bar{D}_{3}=\frac{1}{r_{3}}\left(\bar{C}_{2}-C_{2}\right), \bar{C}_{4}=\frac{1}{r_{4}}\left(\bar{C}_{2}-C_{2}-r_{3} D_{3}\right), \\
& \bar{B}_{3}=\frac{1}{r_{3}{ }^{\prime}}\left(\bar{A}_{2}-A_{2}\right), \bar{A}_{4}=\frac{1}{r_{4}{ }^{\prime}}\left(\bar{A}_{2}-A_{2}-r_{3}{ }^{\prime} B_{3}\right), \\
& \bar{C}_{k}=\frac{1}{r_{k}}\left(\bar{C}_{2}-C_{2}-r_{3} D_{3}-r_{4} C_{4}-\cdots-r_{k-1} D_{k-1}\right), \\
& \bar{A}_{k}=\frac{1}{r_{k}{ }^{\prime}}\left(\bar{A}_{2}-A_{2}-r_{3}{ }^{\prime} B_{3}-r_{4}{ }^{\prime} A_{4}-\cdots r^{\prime}{ }_{k-1} B_{k-1},\right.
\end{aligned}
$$

Substituting into the equation (21), we cbtain finally the following equations.

i.e.

$$
\begin{aligned}
& h_{11} D_{1}+j_{11} B_{1}+g_{12} \bar{C}_{2}+i_{12} \bar{A}_{2}=R_{1}+\Delta_{1} \cdots \cdots \cdots(\mathrm{a} \\
& h_{21} D_{1}+j_{21} B_{1}+g_{22} \bar{C}_{2}+i_{22} \bar{A}_{2}=R_{2}+\Delta_{2} \cdots \cdots \cdots(\mathrm{a} \\
& \left(g_{32}-\frac{1}{r_{3}} h_{33}\right) C_{2}+\left(i_{32}-\frac{1}{\gamma_{3}{ }^{\prime}} j_{33}\right) A_{2}=R_{3}-h_{31} D_{1} \\
& \quad-j_{31} B_{1}-\frac{1}{r_{3}} h_{33} \bar{C}_{2}-\frac{1}{r_{3}{ }^{\prime}} j_{33} \bar{A}_{2}+\Delta_{3} \cdots \cdots \cdots \cdots(
\end{aligned}
$$

therefore in $k$ th equation

$$
\begin{aligned}
& \left(h_{k \cdot k-1}-\frac{\gamma_{k-1}}{r_{k}} g_{k k}\right) C_{k-1}+\left(j_{k \cdot k-1}-\frac{r^{\prime}{ }_{k-1}}{r_{k}{ }^{\prime}} i_{k k}\right) A_{k-1} \\
& =R_{k}+\Delta_{k}-h_{k 1} D_{1}-g_{k 2} C_{2}-h_{k 3} D_{3} \cdots \cdots \\
& -h_{k \cdot k-2} D_{k-2}-\frac{1}{r_{k}} g_{k k}\left(\bar{C}_{2}-C_{2}-r_{3} D_{3}-r_{4} C_{4} \cdots \cdots\right. \\
& \left.-r_{k-2} D_{k-2}\right)-j_{k 1} B_{1}-j_{k 2} A_{2}-j_{k 3} B_{3}-\cdots \cdots \\
& -j_{k \cdot k-2} B_{k-2}-\frac{1}{\gamma_{k}{ }^{\prime}} i_{k k}\left(\bar{A}_{2}-A_{2}-r_{3}{ }^{\prime} B_{3}\right. \\
& \left.-r_{4}{ }^{\prime} A_{4} \cdots \cdots-r_{k-2}^{\prime} B_{k-2}\right)
\end{aligned}
$$

Similary from the equation (22)

$$
\begin{aligned}
& \overline{h^{\prime}}{ }_{11} D_{1}+{\overline{j^{\prime}}}_{11} B_{1}+\overline{g^{\prime}}{ }_{12} \bar{C}_{2}+{\overline{i^{\prime}}}_{12} \bar{A}_{2}=R_{1}{ }^{\prime}+\Delta_{1}{ }^{\prime} \cdots \cdots\left(b_{1}\right) \\
& \overline{h^{\prime}}{ }_{21} D_{1}+\overline{j^{\prime}}{ }_{21} B_{1}+\overline{g^{\prime}}{ }_{22} \bar{C}_{2}+{\overline{i^{\prime}}}_{22} \bar{A}_{2}=R_{2}{ }^{\prime}+\Delta_{2}{ }^{\prime} \cdots \cdots\left(b_{2}\right)
\end{aligned}
$$

in the $k$ th equation

$$
\begin{aligned}
& \left(\bar{h}^{\prime}{ }_{k \cdot k-1}-\frac{\gamma_{k-1}}{\gamma_{k}} \bar{g}^{\prime}{ }_{k k}\right) C_{k-1}+\left(\bar{j}^{\prime}{ }_{k k}-\frac{\gamma_{k-1}{ }^{\prime}}{\gamma_{k}{ }^{\prime}{ }^{\prime}{ }_{k k}}\right) A_{k-1} \\
& =R_{k}{ }^{\prime}+\Delta_{k}{ }^{\prime}-\bar{h}^{\prime}{ }_{k_{1}} D_{1}-\bar{g}^{\prime}{ }_{k_{2}} C_{2}-\bar{h}^{\prime}{ }_{k 3} D_{3} \cdots \cdots \\
& -\bar{h}^{\prime}{ }_{k \cdot k-2} D_{k-2}-\frac{1}{\gamma_{k}} \bar{g}_{k k}{ }_{k}\left(\bar{C}_{2}-C_{2}-r_{3} D_{3}-r_{4} C_{4} \cdots \cdots\right. \\
& \left.-\gamma_{k-2} D_{k-2}\right)-\bar{j}^{\prime}{ }_{k 1} B_{1}-\bar{\imath}^{\prime}{ }_{k 2} A_{2}-\bar{j}^{\prime} k_{3} B_{3} \ldots \ldots
\end{aligned}
$$




$$
\begin{aligned}
& -\bar{j}^{\prime}{ }_{k \cdot k-2} B_{k-2}-\frac{1}{\gamma^{\prime}{ }_{k}} \bar{\imath}_{k k}\left(\bar{A}_{2}-A_{2}-r_{3}{ }^{\prime} B_{3} \cdots \cdots\right.
\end{aligned}
$$

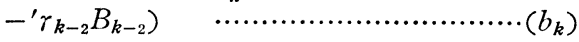

Where $\Delta_{k}$ and $\Delta_{k}{ }^{\prime}(k=1,2,3, \cdots \cdots)$ are residues of difference between $\gamma_{t}, \gamma_{t}{ }^{\prime}$ and $\alpha_{k k+t}, \alpha^{\prime}{ }_{k k+t}$, $\beta_{k k+t}, \beta_{k k+t}^{\prime}$, and also $\Delta_{k}, \Delta_{k}{ }^{\prime}(k=1,2,3)$ are negligible small.

Therefore, from the first four sest of equations, $\left(a_{1}\right),\left(a_{2}\right),\left(b_{1}\right),\left(b_{2}\right)$, we obtain the approximate value of the unknown constant, $D_{1}$, $B_{1}, \bar{C}_{2}$, and $\bar{A}_{2}$, thereby substituting these values into the next two sets of equations $\left(a_{3}\right),\left(b_{3}\right)$, we obtain the approximate value of the unknown constant, $C_{2}, A_{2}$.

And then, we obtain successively the each values of the unknown constant, $D_{k}, B_{k}(k=$ $3,4,5, \cdots \cdots)$ from each two sets of equations $\left(a_{k+1}\right)\left(b_{k+1}\right)$. Again substituting these approximate each value into the first equations (21) and (22), we obtain the more accurate approximate value.

\section{Numerical Example}

We take the example of the skewed plate with two opposite edges simply supported and the other edges free in the case of $a=b, \varepsilon=$ $0.70, \nu=0.30$, full uniform load.

Taking the unknown constant $B_{n}, D_{n}$, to $n=$ 10, we obtain the following Table 1 from the equations (21) and (22).

Table $1_{(\mathrm{a})}$ Array of Coefficients of Eqations (21)

\begin{tabular}{|c|c|c|c|c|c|c|c|c|c|c|c|}
\hline & $j_{k 1}$ & $i_{k 2}$ & $j_{k 3}$ & $i_{k 4}$ & $j_{k 6}$ & $i_{k 6}$ & $j_{k 7}$ & $i_{k 8}$ & $j_{k 9}$ & $i_{k 10}$ & \\
\hline$k=1$ & -0.06355 & 0.01363 & 0.00817 & -0.00316 & -0.00286 & 0.00136 & 0.00145 & -0.00077 & -0.00087 & 0.00049 & \\
\hline$k=2$ & -0.09702 & 0.09802 & 0.07068 & -0.02692 & -0.02397 & 0.01145 & 0.01188 & -0.00632 & -0.00709 & 0.00400 & \\
\hline$k=3$ & -0.05099 & 0.14959 & 0.21239 & -0.09388 & -0.08497 & 0.04039 & 0.04159 & -0.02197 & -0.02453 & 0.01380 & \\
\hline$k=4$ & -0.04537 & 0.12201 & 0.31134 & -0.19847 & -0.20198 & 0.09881 & 0.10218 & -0.05385 & -0.05989 & 0.03355 & \\
\hline$k=5$ & -0.02905 & 0.09899 & 0.30457 & -0.27809 & -0.35446 & 0.19012 & 0.20300 & -0.10801 & -0.12032 & 0.06731 & \\
\hline$k=6$ & -0.02936 & 0.07864 & 0.26509 & -0.29547 & -0.47705 & 0.29775 & 0.34198 & -0.18774 & -0.21174 & 0.11896 & \\
\hline$k=7$ & -0.02038 & 0.06780 & 0.22563 & -0.27620 & -0.52674 & 0.38752 & 0.49625 & -0.28891 & -0.33543 & 0.19103 & \\
\hline$k=8$ & -0.02177 & 0.05706 & 0.19513 & -0.24704 & -0.51835 & 0.43259 & 0.62832 & -0.39700 & -0.48365 & 0.28277 & \\
\hline$k=9$ & -0.01573 & 0.05144 & 0.17040 & -0.21932 & -0.48317 & 0.44328 & 0.71037 & -0.49137 & -0.63804 & 0.38826 & \\
\hline \multirow[t]{2}{*}{$k=10$} & -0.01732 & 0.04480 & 0.15177 & -0.19545 & -0.44122 & 0.42698 & 0.73967 & -0.55609 & -0.77488 & 0.49625 & \\
\hline & $h_{k 1}$ & $g_{k 2}$ & $h_{k 3}$ & $g_{k 4}$ & $h_{k 5}$ & $g_{k 6}$ & $h_{k 7}$ & $g_{k 8}$ & $\boldsymbol{h}_{k 9}$ & $g_{k 10}$ & $R_{k}$ \\
\hline$k=1$ & 0.54295 & -0.20105 & -0.16694 & 0.08192 & 0.08979 & -0.05094 & -0.06115 & 0.03691 & 0.04633 & -0.02892 & 0.018615 \\
\hline$k=2$ & 0.56424 & -0.62243 & -0.56876 & 0.25799 & 0.26399 & -0.14207 & -0.16378 & 0.09575 & 0.11716 & -0.07162 & 0 \\
\hline$k=3$ & 0.31593 & -0.78549 & -1.20500 & 0.60597 & 0.61008 & -0.31698 & -0.35305 & 0.20026 & 0.23877 & -0.14275 & 0.000691 \\
\hline$k=4$ & 0.25352 & -0.63996 & -1.57382 & 1.06137 & 1.16961 & -0.61415 & -0.67527 & 0.37594 & 0.43979 & -0.25327 & 0 \\
\hline$k=5$ & 0.17586 & -0.50851 & -1.51033 & 1.37496 & 1.82685 & -1.03376 & -1.16032 & 0.64554 & 0.74879 & -0.43490 & 0.000150 \\
\hline$k=6$ & 0.16167 & -0.40708 & -1.30673 & 1.42808 & 2.32312 & -1.49616 & -1.78561 & 1.01706 & 1.18650 & -0.68758 & 0 \\
\hline$k=7$ & 0.12237 & -0.34449 & -1.11031 & 1.32723 & 2.50937 & -1.86557 & -2.44788 & 1.46670 & 1.75165 & -1.02418 & 0.000054 \\
\hline$k=8$ & 0.11917 & -0.29277 & -0.95566 & 1.18269 & 2.45184 & -2.05428 & -2.99736 & 1.93088 & 2.40534 & -1.43798 & 0 \\
\hline$k=9$ & 0.09410 & -0.25959 & -0.83345 & 1.04951 & 2.27984 & -2.07536 & -3.32970 & 2.32655 & 3.06891 & -1.90110 & 0.000025 \\
\hline$k=10$ & 0.09454 & -0.22872 & -0.73935 & 0.93371 & 2.07898 & -1.99295 & -3.43859 & 2.59270 & 3.64604 & -2.36560 & 0 \\
\hline
\end{tabular}

Table $1_{(b)}$ Array of Coefficients of Equations (22)

\begin{tabular}{|c|c|c|c|c|c|c|c|c|c|c|c|}
\hline & $j_{k 1}^{\prime}$ & $\bar{i}_{k 2}$ & $j_{k 3}^{\prime}$ & $\bar{i}_{k 4}^{\prime}$ & $j^{\prime}{ }_{k 5}$ & $\bar{i}_{k 6}^{\prime}$ & $j_{k 7}^{\prime}$ & $\bar{i}_{k 8}^{\prime}$ & $j^{\prime}{ }_{k 9}$ & $\bar{i}_{k 1 n}^{\prime}$ & \\
\hline$k=1$ & 0.04820 & -0.01052 & -0.01013 & 0.00487 & 0.00547 & -0.00315 & -0.00383 & 0.00233 & 0.00295 & -0.00185 & \\
\hline$k=2$ & -0.03926 & 0.10102 & 0.08229 & -0.03710 & -0.03848 & 0.02120 & 0.02502 & -0.01496 & -0.01867 & 0.01161 & \\
\hline$k=3$ & 0.04414 & 0.09260 & 0.24378 & -0.12234 & -0.12336 & 0.06416 & 0.07280 & -0.04223 & -0.05154 & 0.03152 & \\
\hline$k=4$ & 0.01036 & -0.03260 & 0.23293 & -0.23739 & -0.27576 & 0.14618 & 0.16242 & -0.09185 & -0.10956 & 0.06574 & \\
\hline$k=5$ & 0.01699 & -0.04251 & 0.02266 & -0.23095 & -0.43107 & 0.26455 & 0.30455 & -0.17203 & -0.20265 & 0.11573 & \\
\hline$k=6$ & 0.00240 & -0.03890 & -0.08026 & -0.08665 & -0.42325 & 0.36456 & 0.47870 & -0.28315 & -0.33731 & - 0.19882 & \\
\hline$k=7$ & 0.00903 & -0.02491 & -0.09772 & 0.03206 & -0.23323 & 0.35983 & 0.60911 & -0.40423 & -0.50589 & 0.30372 & \\
\hline$k=8$ & 0.00036 & -0.02256 & -0.08493 & 0.08051 & -0.02802 & 0.23960 & 0.60318 & -0.48754 & -0.67426 & 0.42507 & \\
\hline$k=9$ & 0.00586 & -0.01439 & -0.07259 & 0.08903 & 0.09537 & 0.08646 & 0.44815 & $-0.48 \Xi 84$ & -0.78322 & 0.53852 & \\
\hline \multirow[t]{2}{*}{$k=10$} & -0.00033 & -0.01433 & -0.05843 & 0.08363 & 0.14623 & -0.02971 & 0.22896 & -0.38598 & -0.77845 & 0.60864 & \\
\hline & $h_{k 1}^{\prime}$ & $\bar{g}^{\prime}{ }_{k 2}$ & $h_{k 3}^{\prime}$ & $\bar{g}_{k 4}^{\prime}$ & $h_{k 5}^{\prime}$ & $\bar{g}_{k 6}^{\prime}$ & $h_{k 7}^{\prime}$ & $\bar{g}_{k 8}^{\prime}$ & $h_{k 9}^{\prime}$ & $\bar{g}_{k 10}^{\prime}$ & $\bar{R}_{k}^{\prime}$ \\
\hline$k=1$ & -0.11252 & 0.02903 & 0.03282 & -0.01722 & -0.01960 & 0.01165 & 0.01523 & -0.00894 & -0.00625 & 0.00813 & -0.008413 \\
\hline$k=2$ & 0.43614 & -0.33148 & -0.27830 & 0.13309 & 0.14339 & -0.08106 & -0.09750 & 0.05911 & 0.07461 & -0.04685 & -0.014177 \\
\hline$k=3$ & 0.03412 & -0.56118 & -0.89705 & 0.44933 & 0.46210 & -0.24761 & -0.28555 & 0.16773 & 0.20677 & -0.12752 & 0.021267 \\
\hline$k=4$ & -0.04000 & -0.15258 & -1.15995 & 0.92388 & 1.05930 & -0.56933 & -0.64125 & 0.36670 & 0.44135 & -0.26678 & -0.007089 \\
\hline$k=5$ & -0.03289 & 0.06528 & -0.54288 & 1.06911 & 1.73068 & -1.04537 & -1.21103 & 0.69013 & 0.81949 & -0.48766 & 0.008862 \\
\hline$k=6$ & -0.02083 & 0.09735 & 0.01387 & 0.65249 & 1.89899 & -1.49203 & -1.92857 & 1.14312 & 1.36951 & -0.81189 & $-0.00472 \tau^{\circ}$ \\
\hline
\end{tabular}




\begin{tabular}{|c|c|c|c|c|c|c|c|c|c|c|c|}
\hline$k=7$ & -0.01899 & 0.08346 & 0.21773 & 0.15236 & 1.34493 & -1.59046 & -2.52504 & 1.65140 & 2.06600 & -1.24467 & 0.005516 \\
\hline$k=8$ & -0.01106 & 0.07231 & 0.25272 & -0.13152 & 0.55431 & -1.23409 & -2.64538 & 2.03944 & 2.78345 & -1.75167 & -0.003546 \\
\hline$k=9$ & -0.01196 & 0.05576 & 0.23876 & -0.24041 & -0.02990 & -0.66533 & -2.17905 & 2.11291 & 3.29823 & $-2,24104$ & 0.003988 \\
\hline$k=10$ & -0.00658 & 0.04897 & 0.20784 & -0.26449 & -0.34188 & -0.16510 & -1.37605 & 1.81351 & 3.39118 & -2.57616 & -0.002836 \\
\hline
\end{tabular}

Assuming the most properble coefficient value $\gamma_{t}, r_{t}{ }^{\prime}$, to the following value, where

$$
\begin{aligned}
& r_{3}=0.90, \quad r_{4}=-0.40, \quad r_{5}=-0.44, \quad r_{6}=0.23, \\
& r_{7}=0.27, \quad r_{8}=-0.18, \quad r_{9}=-0.22, \quad r_{10}=0.14, \\
& \text { and } r_{3}{ }^{\prime}=0.80, \quad r_{4}{ }^{\prime}=-0.30, \quad r_{5}{ }^{\prime}=-0.30, \\
& r_{6}{ }^{\prime}=0.15, \quad r_{7}^{\prime}=0.18, \quad r_{8}{ }^{\prime}=-0.10, \\
& r_{9}{ }^{\prime}=-0.12, \quad r_{10}{ }^{\prime}=0.077,
\end{aligned}
$$

we have the first approximate value from $\left(a_{1}\right)$, $\left(a_{2}\right), \cdots \cdots\left(a_{10}\right)$, and $\left(b_{1}\right),\left(b_{2}\right), \cdots \cdots\left(b_{10}\right)$. i.e.

$$
\begin{array}{ll}
B_{1}{ }^{(1)}=-0.19446, & D_{1}^{(1)}=0.01943, \\
\bar{A}_{2}{ }^{(1)}=-0.29534, & \bar{C}_{2^{(1)}}=0.001344, \\
A_{2}{ }^{(1)}=-0.57925, & C_{2}{ }^{(1)}=-0.005428, \\
B_{3}{ }^{(1)}=0.66492, & D_{3}^{(1)}=0.03506, \\
A_{4}{ }^{(1)}=3.24861, & C_{4}{ }^{(1)}=0.74938, \\
B_{5}{ }^{(1)}=-4.19376, & D_{5}{ }^{(1)}=-1.17940, \\
A_{6}{ }^{(1)}=-4.81379, & C_{6}{ }^{(1)}=-1.35297, \\
B_{7}{ }^{(1)}=1.47483, & D_{7^{(1)}}=0.30354, \\
A_{8}{ }^{(1)}=1.11620, & C_{8}{ }^{(1)}=0.19116, \\
B_{9}{ }^{(1)}=-0.47793, & D_{9}{ }^{(1)}=-0.15862,
\end{array}
$$

Substituting these value into the equation (24), we have the approximate value of $\bar{B}_{3}, \bar{D}_{3}, \ldots \ldots$ $\bar{A}_{10}, \bar{C}_{10}$.

$$
\begin{aligned}
& \text { i.e. } \bar{B}_{3}{ }^{(1)}=0.35489, \quad \bar{D}_{3}{ }^{(1)}=0.00751 \text {, } \\
& \bar{A}_{4}^{(1)}=0.82666, \quad \bar{C}_{4}^{(1)}=0.06207 \text {, } \\
& \bar{B}_{5}^{(1)}=-2.42200, \quad \bar{D}_{5}^{(1)}=-0.62483 \text {, } \\
& \bar{A}_{6}{ }^{(1)}=-3.54391, \quad \bar{C}_{6}{ }^{(1)}=-1.06180 \text {, } \\
& \bar{B}_{7}^{(1)}=1.05831 . \quad \bar{D}_{7^{(1)}}=0.24909 \text {, } \\
& \bar{A}_{8}{ }^{(1)}=0.74968, \quad \bar{C}_{8}{ }^{(1)}=0.08178 \text {, } \\
& \bar{B}_{9}^{(1)}=-0.30594, \quad \bar{D}_{9}^{(1)}=0.08943 \text {, } \\
& \bar{A}_{10}{ }^{(1)}=-0.26735, \quad \bar{C}_{10}{ }^{(1)}=-0.10808
\end{aligned}
$$

\begin{tabular}{|c|c|c|c|c|c|c|c|}
\hline $\begin{array}{l}\text { Lo- } \\
\text { cation }\end{array}$ & $M_{y}$ & $M_{x}$ & $M_{x y}$ & $\begin{array}{l}\text { Lo- } \\
\text { cation }\end{array}$ & $M_{y}$ & $M_{x}$ & $M_{x y}$ \\
\hline$a_{1}$ & 0.12791 & 0.06366 & 0.04669 & $d_{3}$ & $0.28662 \mid$ & $|-0.09258|$ & 0.08093 \\
\hline$a_{2}$ & 0.20806 & 0.11015 & 0.09475 & $d_{4}$ & 0.28613 & 0.05804 & 0.14578 \\
\hline$a_{3}$ & 0.25415 & 0.14006 & 0.12792 & $d_{5}$ & 0.29501 & 0.06623 & 0.17618 \\
\hline$a_{4}$ & 0.26886 & 0.14726 & 0.13996 & $d_{6}$ & 0.17371 & 0.13176 & 0.18779 \\
\hline$c_{1}$ & 0.14657 & -0.02468 & 0.01717 & $d_{7}$ & 0.06889 & 0.11498 & 0.10529 \\
\hline$c_{2}$ & 0.27281 & 0.00780 & 0.09021 & $e_{3}$ & 0.28276 & -0.10393 & 0.14592 \\
\hline$c_{3}$ & 0.27468 & 0.09179 & 0.10612 & $e_{4}$ & 0.32166 & 0.03247 & 0.15942 \\
\hline$c_{4}$ & 0.29975 & 0.10383 & 0.14668 & $e_{5}$ & 0.28111 & 0.10531 & 0.18806 \\
\hline$c_{5}$ & 0.26609 & 0.11972 & 0.15970 & $e_{6}$ & 0.25188 & 0.08735 & 0.26012 \\
\hline$c_{6}$ & 0.18585 & 0.12821 & 0.12100 & $e_{7}$ & 0.07847 & 0.12220 & 0.16133 \\
\hline$c_{7}$ & 0.09732 & 0.08913 & 0.06909 & & & & \\
\hline
\end{tabular}

\begin{tabular}{|c|c|c|c|c|c|c|c|}
\hline $\begin{array}{l}\text { Lo- } \\
\text { cation }\end{array}$ & $M_{n}$ & $M_{t}$ & $\alpha$ & $\left|\begin{array}{c}\text { Lo- } \\
\text { cation }\end{array}\right|$ & $M_{n}$ & $M_{t}$ & $\alpha$ \\
\hline$a_{1}$ & 0.15224 & 0.03914 & $27^{\circ} 45^{\prime}$ & $d_{3}$ & 0.30318 & $-0.10914 \mid$ & $11^{\circ} 35^{\prime}$ \\
\hline$a_{2}$ & 0.26576 & 0.05246 & $31^{\circ} 20^{\prime}$ & $d_{4}$ & 0.35627 & $-0.01209 \mid$ & $26^{\circ} 0^{\prime}$ \\
\hline$a_{3}$ & 0.33720 & 0.05700 & $33^{\circ} 0$ & $d_{5}$. & 0.39068 & $-0.02943 \mid$ & $28^{\circ} 30^{\prime}$ \\
\hline$a_{4}$ & 0.36071 & 0.05541 & $33^{\circ} 15^{\prime}$ & $d_{6}$ & 0.34180 & -0.03633 & $41^{\circ} 50^{\prime}$ \\
\hline$c_{1}$ & 0.14109 & -0.01920 & $5^{\circ} 40^{\prime}$ & $d_{7}$ & 0.19958 & -0.01572 & $38^{\circ} 20^{\prime}$ \\
\hline$c_{2}$ & 0.30082 & -0.02025 & $17^{\circ} 15^{\prime}$ & $e_{3}$ & 0.33192 & -0.15308 & $18^{\circ} 0^{\prime}$ \\
\hline$c_{3}$ & 0.32334 & 0.04313 & $24^{\circ} 40^{\prime}$ & $e_{4}$ & 0.38922 & -0.03508 & $23^{\circ} 30^{\prime}$ \\
\hline$c_{4}$ & 0.37679 & 0.02619 & $28^{\circ} 5^{\prime}$ & $e_{5}$ & 0.40076 & -0.01432 & $31^{\circ} 50^{\prime}$ \\
\hline$c_{5}$ & 0.36790 & 0.01792 & $32^{\circ} 45^{\prime}$ & $e_{6}$ & 0.44312 & -0.10388 & $36^{\circ} 15^{\prime}$ \\
\hline$c_{B}$ & 0.28123 & 0.03283 & $38^{\circ} 20^{\prime}$ & $e_{7}$ & 0.26313 & -0.06246 & $41^{\circ} 8^{\prime}$ \\
\hline$c_{7}$ & $|0.16248|$ & 0.02398 & $43^{\circ} 20^{\prime}$ & & & & \\
\hline
\end{tabular}

Therefore, substituting these value $B_{1}, D_{1}, C_{2}$, $D_{3}, \cdots \cdots$ and $\bar{A}_{k}, \bar{C}_{k}$ into the first equations (21) and (22), we get the secondary approximate value $B_{k}{ }^{(2)}, D_{k}{ }^{(2)}, \cdots \cdots$, i.e. we get the value of $D_{1}{ }^{(2)}$ from the first equation $\left(a_{1}\right)$ of $(21)$, and $B_{1}{ }^{(2)}$ from the first equation $\left(b_{1}\right)$ of $(22)$, and then successively we get the value of $C_{k}{ }^{(2)}$ from the $k$ th equation $\left(a_{k}\right)$, and $A_{k}{ }^{(2)}$ from the $k$ th equation $\left(b_{k}\right)$.

Thereby, these values are as follows.

$$
\text { i.e. } \begin{array}{ll}
B_{1}{ }^{(2)}=-0.18041, & D_{1}{ }^{(2)}=0.02267, \\
A_{2}{ }^{(2)}=-0.54949, & C_{2}{ }^{(2)}=-0.00443, \\
B_{3}{ }^{(2)}=0.69287, & D_{3}{ }^{(2)}=0.04781, \\
A_{4}{ }^{(2)}=3.49605, & C_{4}{ }^{(2)}=0.76078, \\
B_{5}{ }^{(2)}=-4.34633, & D_{5}{ }^{(2)}=-1.12583,
\end{array}
$$

$$
\begin{array}{ll}
A_{6}{ }^{(2)}=-5.17175, & C_{6}{ }^{(2)}=-1.36515, \\
B_{7}{ }^{(2)}=1.55240, & D_{7}{ }^{(2)}=0.32658, \\
A_{8}{ }^{(2)}=1.05188, & C_{8}{ }^{(2)}=0.18154, \\
B_{9}{ }^{(2)}=-0.50276,, & D_{9}{ }^{(2)}=-0.17457,
\end{array}
$$

As these value equals nearly the first value, we can consider them as a true value.

Therefore, substituting the above-mentioned value into the expression of moment, we obtain the following table.

Table 2 Moment of Uniformly Loaded Skewed Plate.

From the Table 2, the maximum moment and the direction is obtained as follows.

From the Table 3, we obtain the following principal moment diagram.

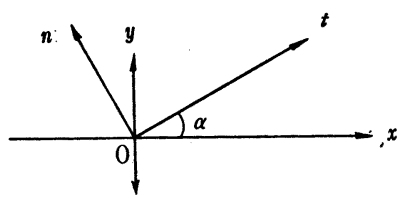

Fig. $3_{(a)}$ Indicates the location of $x, y$ in the each point of moment, shear, and reaction. 


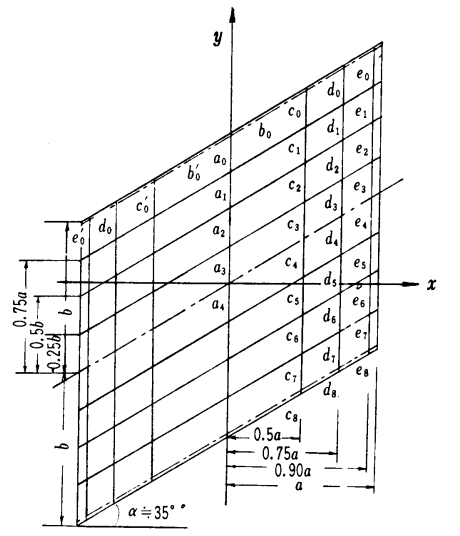

Fig. $3_{(b)}$

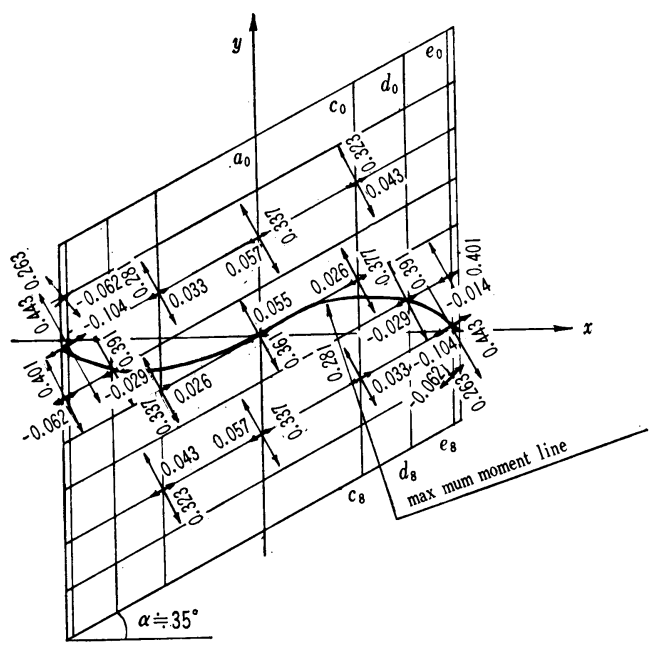

Fig. 4 Principal Moment Diagram Coeff. $p a^{2}$.

Similary, substituting the above-mentioned value into the expression of shear, we obtain the following table.

Table 4 Shear of:Uniformly Loaded Skewed Plate.

\begin{tabular}{|c|c|c|c|c|c|}
\hline Location & $Q_{y}$ & $Q_{x}$ & Location & $Q_{y}$ & $Q_{x}$ \\
\hline$a_{0}$ & 0.63452 & -0.44434 & $d_{2}$ & 0.38184 & -0.20717 \\
\hline$a_{1}$ & 0.48174 & -0.32560 & $d_{3}$ & 0.19033 & -0.10954 \\
\hline$a_{2}$ & 0.32422 & -0.21299 & $d_{4}$ & 0.11580 & -0.01145 \\
\hline$a_{3}$ & 0.16315 & -0.10600 & $d_{5}$ & -0.08518 & 0.13523 \\
\hline$a_{4}$ & 0 & 0 & $d_{6}$ & -0.29076 & 0.22750 \\
\hline$c_{0}$ & 0.69382 & -0.48563 & $d_{7}$ & -0.46803 & 0.33892 \\
\hline$c_{1}$ & 056761 & -0.33240 & $d_{8}$ & -0.64514 & 0.45270 \\
\hline$c_{2}$ & 0.34406 & -0.21328 & $e_{3}$ & 0.21538 & -0.13385 \\
\hline$c_{3}$ & 0.16818 & -0.04806 & $e_{4}$ & 0.17983 & -0.03385 \\
\hline$c_{4}$ & 0.02764 & 0.03770 & $e_{5}$ & -0.05725 & 0.10425 \\
\hline$c_{5}$ & -0.13384 & 0.12732 & $e_{6}$ & -0.21427 & 0.21259 \\
\hline$c_{6}$ & -0.31178 & 0.23105 & $e_{7}$ & -0.47197 & 0.32313 \\
\hline$c_{7}$ & -0.48046 & 0.34061 & $e_{8}$ & -0.61383 & 0.45183 \\
\hline$c_{8}$ & -0.64972 & 0.45499 & & & \\
\hline
\end{tabular}

Furthermore, substituting into the expression of reaction, we obtain the following table.

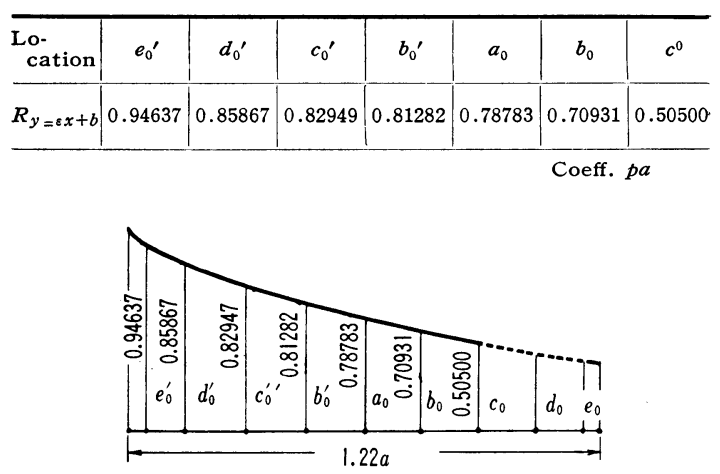

Fig. 5 Distribution of Reaction.

Table 5 indicates the numerical results of the square plate under the uniform load, comparing these values with the numerical ones for the skewed plate.

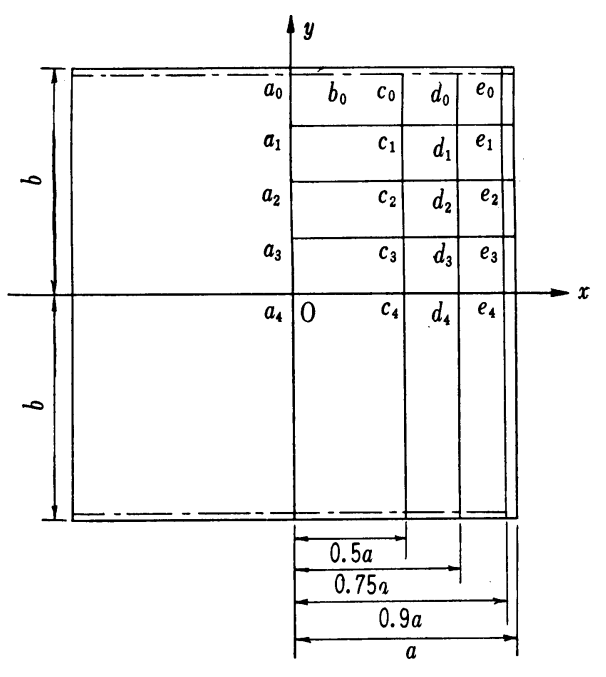

Fig. 6 Indicates the location of $x, y$ in each point of moment, shear, and reaction.

Table $5_{(a)}$ Moment of Uniformly Loded Square Plate.

\begin{tabular}{c|c|c|c|c|c|c|c}
\hline $\begin{array}{c}\text { Lo- } \\
\text { cation }\end{array}$ & $M_{y}$ & $M_{x}$ & $M_{x y}$ & $\begin{array}{c}\text { Lo- } \\
\text { cation }\end{array}$ & $M_{y}$ & $M_{x}$ & $M_{x y}$ \\
\hline$a_{1}$ & 0.21341 & 0.05121 & 0 & $d_{0}$ & 0 & 0 & 0.05251 \\
$a_{2}$ & 0.36515 & 0.08591 & 0 & $d_{1}$ & 0.21850 & 0.03079 & 0.04496 \\
$a_{3}$ & 0.45497 & 0.10558 & 0 & $d_{2}$ & 0.37448 & 0.04819 & 0.03713 \\
$a_{4}$ & 0.48606 & 0.11237 & 0 & $d_{3}$ & 0.46716 & 0.05631 & 0.02009 \\
$c_{0}$ & 0 & 0 & 0.02828 & $d_{4}$ & 0.49926 & 0.05902 & 0 \\
$c_{1}$ & 0.21531 & 0.04292 & 0.02613 & $e_{0}$ & 0 & 0 & 0.07355 \\
$c_{2}$ & 0.36864 & 0.07077 & 0.02000 & $e_{1}$ & 0.22166 & 0.01975 & 0.06794 \\
$c_{3}$ & 0.45984 & 0.08554 & 0.01082 & $e_{2}$ & 0.38038 & 0.02772 & 0.05194 \\
$c_{4}$ & 0.49101 & 0.09066 & 0 & $e_{3}$ & 0.47489 & 0.02958 & 0.02815 \\
& & & & $e_{4}$ & 0.50761 & 0.02884 & 0 \\
\hline
\end{tabular}


Table $5_{(b)}$ Shear of Uniformly Loadəd Square Plate.

\begin{tabular}{c|c|c|c|c|c}
\hline Location & $Q_{y}$ & $Q_{x}$ & Location & $Q_{y}$ & $Q_{x}$ \\
\hline$a_{0}$ & 0.93678 & 0 & $d_{0}$ & 0.88919 & 0 \\
$a_{1}$ & 0.69242 & 0 & $d_{1}$ & 0.64763 & 0.03504 \\
$a_{2}$ & 0.45593 & 0 & $d_{2}$ & 0.42165 & 0.06473 \\
$a_{3}$ & 0.22615 & 0 & $d_{3}$ & 0.20760 & 0.08458 \\
$a_{4}$ & 0 & 0 & $d_{4}$ & 0 & 0.09155 \\
$c_{6}$ & 0.91745 & 0 & $e_{0}$ & 0.86433 & 0 \\
$c_{1}$ & 0.67373 & 0.02070 & $e_{1}$ & 0.62464 & 0.04609 \\
$c_{2}$ & 0.44163 & 0.03825 & $e_{2}$ & 0.40406 & 0.08517 \\
$c_{3}$ & 0.21841 & .0 .04998 & $e_{3}$ & 0.19807 & 0.11128 \\
$c_{4}$ & 0 & 0.05410 & $e_{4}$ & 0 & 0.12044 \\
\hline \multicolumn{1}{c}{} & \multicolumn{3}{|c}{ Coeff. pa }
\end{tabular}

and then, the reaction is expressed as follows.

Table $5_{(c)}$ Distribution of Reaction

\begin{tabular}{c|c|c|c|c|c}
\hline Location & $a_{5}$ & $b_{5}$ & $c_{0}$ & $d_{0}$ & $e_{0}$ \\
\hline$R)_{y=b}$ & 0.88949 & 0.87741 & 0.83874 & 0.76575 & 0.69977 \\
\hline \multicolumn{4}{c}{ Coeff. $p a$}
\end{tabular}

\section{Conclusion}

The calculation of the skewed plate is in the case of full uniform load, but this can apply in the case of any load and any skew angle of the plate.

And this solution can not only to the other skewed plate with two opposite edges simply supported and the other edges various conditions, but can apply to a rectangular plate with any boundary condition.

Acknowledgement. The author wishes to express his indebtedness to the scientific research fund from the Ministry of Education of Japan for a part of the expense of the analysis of the skewed plate.

\section{References}

1) W. Fuchsteiner, : Entwicklungs functionen für polygonal begrenzte dünne platten (Der Bauingenieur, 1953, Heft 7)

2) Homberg, Marx, Schiefe Stäbe und Platten.

（原稿受付 : 1961.3.29） 